\title{
Progress risk assessment of oral premalignant lesions with saliva miRNA analysis
}

\author{
Ya Yang ${ }^{1}$, Yue-xiu Li ${ }^{2}, X_{i}$ Yang $^{3}$, Long Jiang ${ }^{1}$, Zuo-jun Zhou ${ }^{1}$ and Ya-qin Zhu ${ }^{*}$
}

\begin{abstract}
Background: Oral cancer develops through multi-stages: from normal to mild (low grade) dysplasia (LGD), moderate dysplasia, and severe (high grade) dysplasia (HGD), to carcinoma in situ (CIS) and finally invasive oral squamous cell carcinomas (OSCC). Clinical and histological assessments are not reliable in predicting which precursor lesions will progress. The aim of this study was to assess the potential of a noninvasive approach to assess progress risk of oral precancerous lesions.

Methods: We first used microRNA microarray to profile progressing LGD oral premaligant lesions (OPLs) from nonprogressing LGD OPLs in order to explore the possible microRNAs deregulated in low grade OPLs which later progressed to HGD or OSCC. We then used RT-qPCR to detect miRNA targets from the microarray results in saliva samples of these patients.
\end{abstract}

Results: We identified a specific miRNA signature that is aberrantly expressed in progressing oral LGD leukoplakias. Similar expression patterns were detected in saliva samples from these patients.

Conclusions: These results show promise for using saliva miRNA signature for monitoring of cancer precursor lesions and early detection of disease progression.

Keywords: Oral leukoplakia, Malignant transformation, Risk assessment, miRNAs, Salivary biomarker

\section{Background}

Oral squamous cell carcinomas (OSCCs) are among the most common types of head and neck cancers and are a major cause of significant morbidity. It was reported that $16-62 \%$ of OSCCs develop from premalignant lesions [1], which often presents clinically as white or red mucosal patches called leukoplakia and erythroplakia. Early detection of cancer development from oral premalignant lesions (OPLs) plays a crucial role in successful therapy. Currently risk of progression in oral leukoplakia is typically determined based on clinical assessment and histopathological evaluation of biopsied material. High grade dysplasia (HGD) and carcinoma in situ (CIS) are considered to have a high risk for progression to invasive disease. In contrast, most of the low grade dysplasias (LGDs) remain unchanged for years or even resolve over

\footnotetext{
*Correspondence: julieyy@163.com

'Department of General Dentistry, Ninth People's Hospital, School of Stomatology, Shanghai Jiao Tong University School of Medicine,Shanghai Key Laboratory of Stomatology, 639 Zhi Zao Ju Road, Shanghai 200011, China

Full list of author information is available at the end of the article
}

time [2]. But a small proportion of these LGDs may progress to carcinomas [3]. Clinical and histological characteristics cannot be used to separate "progressing" and "non-progressing" LGDs [4]. There is therefore an urgent need to find predictive biomarkers that can aid in defining progression likelihood of LGDs, which represent the majority of diagnosed OPLs.

miRNAs are an abundant class of small 18-25 nucleotides long single-stranded non-coding RNA. These noncoding RNAs participate in a variety of biologic processes including development, differentiation, apoptosis and proliferation through regulating its target genes [5-8]. Notably, a single miRNA is capable of regulating the translation of a multitude of genes [9]. And they are remarkably stable both in saliva samples and in tissue samples $[10,11]$, which offers a great advantage over other classes of biomarkers and also an extremely important characteristic in clinical settings. The control of gene expression by miRNAs is a process seen in virtually all cancer cells. Recently, a bundle of studies have showed that miRNAs might behave as cancer 'drivers'

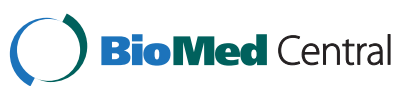


and the aberrant expressions could profoundly contribute to the progression from premalignant lesion to cancer [12-14]. Only a small number of studies have investigated miRNA expression profiles in the malignant transformation of OPLs, and there is no report yet about investigating salivary miRNAs changes in this type of patients. Cervigne et al. examined miRNA expression changes in tissue samples during progression of oral malignancy [15]. Wiklund et al. demonstrates that Certain OSCC specific miRNA are both present and detectable in oral fluids [16]. However salivary miRNA changes involved in the transformation of oral leukoplakias remain poorly understood.

With an aim to investigate the potential of using salivary microRNA biomarkers to assess the risk of malignant transformation, our study includes the analysis of progressing and non-progressing LGD leukoplakias with similar histopathological diagnosis but different clinical outcomes. We first used microRNA microarray to profile progressing LGDs from non-progressing LGDs. We identified a specific miRNA signature that is aberrantly expressed in oral LGD leukoplakias which later progressed to HGDs or CISs and even OSCCs. Similar miRNA deregulation patterns were detected in saliva samples of these patients. These results indicated the feasibility of a noninvasive assay for risk assessment of oral precancerous lesions based on salivary miRNA signatures.

\section{Methods}

\section{Patients and samples collection}

From 2006 to 2012, 45 eligible patients treated in the department of Oral \& Maxillofacial-Head \& Neck Oncology, Ninth People's Hospital, Shanghai Jiao Tong University (Shanghai, China) were enrolled in the present study. The patients had been diagnosed with LGD leukoplakias and were given 13-cis-retinoic acid (13cRA) treatment and close follow-ups before any event (defined as the diagnosis of HGD or CIS or OSCC). The diagnosis of leukoplakias, OSCC, and LGD were made according to World Health Organization criteria [17]. A total of 45 frozen samples were collected at baseline after enrollment. Among these 45 LGD leukoplakias patients, 10 patients progressed to carcinoma in situ or OSCC after an average time of 30.1 months. 2 were excluded because of poor RNA quality. Among the 35 patients who did not develop OSCC, 5 were excluded because of poor RNA quality, and another 12 were excluded because of new lesion occurrence. As these progressing LGD samples are very rare and difficult to obtain, we included all the 8 samples from patients who developed oral cancer $(\mathrm{N}=8)$ for gene expression profiling. 7 patients who remained at a LGD stage within 3-5 years of follow-up were selected, in order to identify the differences in miRNA expression between these precancerous lesions with different clinical outcomes. Informed consent was obtained from all patients. The project was approved by the Scientific and Ethics Committee of Shanghai Jiao Tong University. Patient information and representative histological pictures is shown in Table 1 and Figure 1, respectively.

Saliva samples were collected from these patients whenever tissue biopsies were collected. But only samples collected at first visit were used for analysis in order to investigate miRNA expression differences in these premalignant lesions at LGD stage. Saliva samples from 7 healthy volunteers were also collected to determine baseline miRNA expression in oral saliva from normal control. Briefly, immediately after mouthwash, $\sim 2 \mathrm{ml}$ saliva was collected. Upon collection, saliva samples were mixed with $5 \mathrm{ml}$ RNA Protect Saliva reagent (Qiagen, Copenhagen), kept at room temperature for 24 hours, and then stored at $-20^{\circ} \mathrm{C}$ until RNA or protein isolation.

\section{RNA extraction, CDNA synthesis and miRNA expression profiling for tissue samples}

The TaqMan $^{\circledR}$ low density array (TLDA) qRT-PCR system (Applied Biosystems, Foster City CA) were used for global miR expression analysis. Briefly, total RNA was extracted using TRIzol reagent (Invitrogen).The qualities of RNA extracts were found to be good. Total RNA was converted into specific cDNA derived from mature miRs and quantified using Multiplex RT and TaqMan Low Density Array (TLDA) Human MicroRNA Panel v3.0 and 7900 real-time RT PCR System (Applied Biosystems). A total of 800 ng RNAs were used for each array. The two-card set of TaqMan ${ }^{\circledR}$ Array MicroRNA Cards (Cards A and B) contained 754 lyophilized human TaqMan miR sequences. And each card contains four control assays-three carefully selected candidate endogenous control assays and one negative control assay.

\section{RNA extraction, cDNA synthesis and miRNA quantification for saliva samples}

Real-time qPCR analysis was performed on saliva samples from these patients and 7 healthy volunteers using the TaqMan MicroRNA Assay. miRNA expression analysis was performed for 8 selected deregulated miRNAs (miR-10b, miR-145, miR-99b, miR-708, miR-181c, miR30e, miR-660 and miR-197) with greatest fold changes in progressing LGD leukoplakias compared with nonprogressing LGD leukoplakias.

Total RNA was extracted from saliva samples using the RNeasy Micro Kit (Qiagen, Hilden, Germany) according to the manufacturers' protocols. In brief, the saliva sample is first centrifuged, and Buffer RLT is then added to the resulting pellet. After addition of ethanol, the sample is applied to an RNeasy MinElute spin column, where RNA 
Table 1 Sample set of oral LGD leukoplakias

\begin{tabular}{|c|c|c|c|c|c|c|}
\hline Patients & Lesion site & $\begin{array}{l}\text { Histopathological } \\
\text { Diagnosis }\end{array}$ & Age & Sex & $\begin{array}{l}\text { Tobacco } \\
\text { Exposure }\end{array}$ & $\begin{array}{l}\text { Duration of } \\
\text { follow-up(mo) }\end{array}$ \\
\hline 1 & Left lateral tongue & Mild to moderate dysplasia & 45 & female & former smoker & 40 \\
\hline 2 & Right lateral palatine & Mild to moderate dysplasia & 67 & male & current smoker & 36 \\
\hline 3 & Right lateral palatine & Mild to moderate dysplasia & 55 & female & never smoked & 39 \\
\hline 4 & right lateral tongue & Mild to moderate dysplasia & 54 & female & never smoked & 36 \\
\hline 5 & right cheek mucosa & Mild to moderate dysplasia & 64 & male & current smoker & 43 \\
\hline 6 & right cheek mocusa & Mild to moderate dysplasia & 57 & male & current smoker & 46 \\
\hline 7 & left cheek mucosa & Mild dysplasia & 61 & male & current smoker & 39 \\
\hline 8 & left lateral tongue & Severe dysplasia SCC micro-foci & 71 & male & current smoker & 29 \\
\hline 9 & lower lip & Severe dysplasia, SCC micro-foci & 60 & female & never smoked & 28 \\
\hline 10 & left cheek & Severe dysplasia SCC micro-foci & 52 & female & former smoker & 34 \\
\hline 11 & palate & Severe dysplasia & 55 & female & never smoked & 35 \\
\hline 12 & left cheek & Severe dysplasia & 66 & male & former smoker & 30 \\
\hline 13 & right lateral tongue & carcinoma in situ & 61 & female & never smoked & 29 \\
\hline 14 & right lateral tongue & carcinoma in situ & 71 & male & current smoke & 30 \\
\hline 15 & left cheek & moderate to severe dysplasia SCC micro-foci & 59 & male & current smoker & 26 \\
\hline
\end{tabular}

binds to the membrane after centrifugation. Traces of genomic DNA are removed by DNase digestion, and contaminants are washed away in several wash steps. Highly pure RNA is then eluted using RNase-free water in a volume of just $14 \mu \mathrm{l}$. The purity of isolated RNA was determined by OD260/280 using a Nanodrop ND-1000 (Thermo Scientific, Worcester, MA). Each RNA sample was polyadenylated and reversely transcribed to cDNA using the TaqMan ${ }^{\circledR}$ MicroRNA Reverse Transcription Kit (Applied Biosystems, Foster City, CA Single-stranded cDNA was synthesized from $10 \mathrm{ng}$ of total RNA(normalization by amount) or from $1 \mu \mathrm{l}$ of the RNA volume (normalization by volume) using specific miRNA primers (TaqMan MicroRNA Assay, PN 4427975, Applied Biosystems).

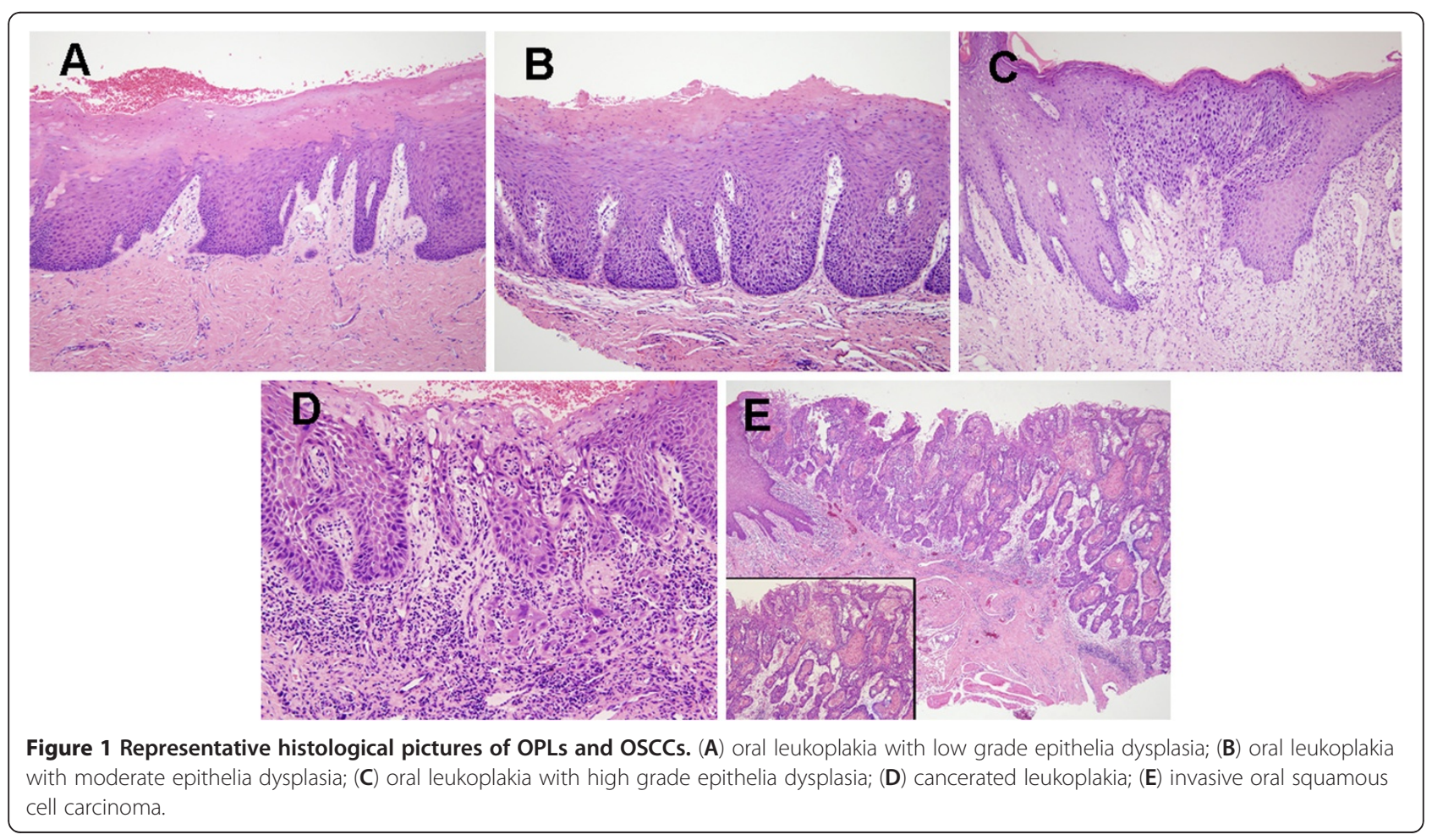


All assays were performed in duplicate. Expression data were normalized against RNU6; pooled saliva samples from healthy volunteers were used as the reference for analysis.

\section{Statistical analyses}

Samples were grouped as normal, non-progressive LGD leukoplakia and progressive LGD leukoplakia. Data were quantified and analyzed using Sequence Detection System (version 2.3) (Applied Biosystems). The expression level of each miRNA was quantified by its normalized threshold cycle number $\Delta \mathrm{Ct}$, where $\Delta \mathrm{Ct}=[\mathrm{Ct}$ (miRNA)]-[Ct (U48)], and the relative expression level was calculated as $2^{-(\Delta C t)}$ which is commonly used in genom-wide profiling studies of miRNAs. In order to select differentially expressed miRNAs for further classification, the random variance t-test (RVM t-test) was used to compare the control and experiment, as the RVM t-test can raise degrees of freedom effectively in the cases of small samples. The multiple testing was corrected using the BenjaminiHochberg false discovery rate (FDR) method. Having a differential fold change $> \pm 2$ at raw $P$ value $<0.05$ were considered as significant candidates.

Expression levels of selected miRNAs in saliva samples of these patients were calculated with U6 as normalization factor. Comparison of raw U6 values between different groups showed no significant difference ( $\mathrm{p}=0.09$, MannWhitney $U$ test). Raw data were normalized by subtracting the U6 CT values from the marker CT values. Statistical comparisons were made with the use of student's t-tests for two groups. Data analyses were performed using SPSS for Windows version 16.0 (SPSS Inc., USA). Tests were two-sided and $\mathrm{p} \leq 0.05$ was considered statistically significant.

\section{Results}

miRNA expression profiling in different types of LGD lesions

In order to evaluate miRNA deregulations of progressive oral premalignant lesions, we used a systematic miRNA expression profiling analysis of 754 mammalian miRNAs

Table 2 miRNAs differentially expressed between progressing-LGD leukoplakias and non-progressing-LGD leukoplakias

\begin{tabular}{|c|c|c|c|c|c|}
\hline \multirow[b]{2}{*}{ miRNA } & \multirow{2}{*}{$\begin{array}{l}\text { Mean intensity in } \\
\text { Non-progressing LGDs }\end{array}$} & \multicolumn{4}{|l|}{ Mean intensity in } \\
\hline & & Progressing LGDs & Fold change & Unadjusted $p$ value & FDR \\
\hline miR-10b-5p & 10.2689 & 13.7939 & $11.51 \uparrow$ & 0.008 & 0.069 \\
\hline miR-99a-5p & 10.9423 & 8.1768 & $6.80 \downarrow$ & 0.011 & 0.069 \\
\hline miR-99b-5p & 16.3981 & 12.1692 & $18.75 \downarrow$ & 0.003 & 0.016 \\
\hline$m i R-145-5 p$ & 13.3692 & 9.6968 & $12.75 \downarrow$ & 0.011 & 0.069 \\
\hline miR-100-5p & 11.9107 & 8.8512 & $8.33 \downarrow$ & 0.011 & 0.0698 \\
\hline miR-125b-5p & 10.9423 & 8.1768 & $6.80 \downarrow$ & 0.011 & 0.0698 \\
\hline miR-181b & 9.9163 & 6.1030 & $14.05 \downarrow$ & 0.016 & 0.069 \\
\hline miR-181c & 20.6273 & 16.6825 & $15.40 \downarrow$ & 0.011 & 0.069 \\
\hline miR-197-3p & 16.4423 & 12.3639 & $16.89 \downarrow$ & 0.042 & 0.128 \\
\hline miR-331-3p & 10.9681 & 8.1938 & $6.84 \downarrow$ & 0.010 & 0.069 \\
\hline miR-15a-5p & 16.5175 & 12.8715 & $12.51 \downarrow$ & 0.002 & 0.056 \\
\hline miR-708 & 8.8610 & 12.4334 & $11.89 \uparrow$ & 0.014 & 0.069 \\
\hline miR-150-5p & 7.9801 & 5.4854 & $5.63 \downarrow$ & 0.002 & 0.0567 \\
\hline miR-30e-3p & 6.6441 & 10.9956 & $20.41 \uparrow$ & 0.002 & 0.056 \\
\hline miR-30a-3p & 15.2221 & 17.1464 & $3.80 \uparrow$ & 0.049 & 0.985 \\
\hline miR-21 & 7.1456 & 9.5243 & $5.20 \uparrow$ & 0.049 & 0.985 \\
\hline let-7a-5p & 20.3130 & 17.7931 & $5.74 \downarrow$ & 0.039 & 0.985 \\
\hline$m i R-335-5 p$ & 9.2299 & 10.2837 & $2.08 \uparrow$ & 0.039 & 0.995 \\
\hline miR-144* & 11.5283 & 15.1400 & $12.22 \uparrow$ & 0.005 & 0.057 \\
\hline miR-25-3p & 8.8177 & 12.5057 & $12.88 \uparrow$ & 0.017 & 0.069 \\
\hline miR-19a-3p & 5.3255 & 8.5336 & $9.24 \uparrow$ & 0.007 & 0.066 \\
\hline miR-660-5p & 7.8916 & 12.6950 & $27.92 \uparrow$ & 0.020 & 0.076 \\
\hline miR-140-5p & 11.1943 & 15.2157 & $16.23 \uparrow$ & 0.012 & 0.069 \\
\hline$m i R-590-5 p$ & 7.4825 & 11.4638 & $15.79 \uparrow$ & 0.004 & 0.057 \\
\hline miR-9 & 21.1533 & 17.9955 & $8.92 \downarrow$ & 0.039 & 0.915 \\
\hline
\end{tabular}


on 8 progressing LGD leukoplakias and 7 non-progressing LGD leukoplakias. A filter procedure reduced the number of miRNAs to a total of 617 hsa-miRNAs, eliminating genes with low expression variation across the experiments $(\mathrm{SD}<0.3)$. We performed a differential expression analysis to detect significant differences in miRNA expression between different types of LGD leukoplakias.

We identified 25 miRNAs differentially expressed between progressive and non-progressive LGD leukoplakias. Compared to non-progressive LGD leukoplakias, 13

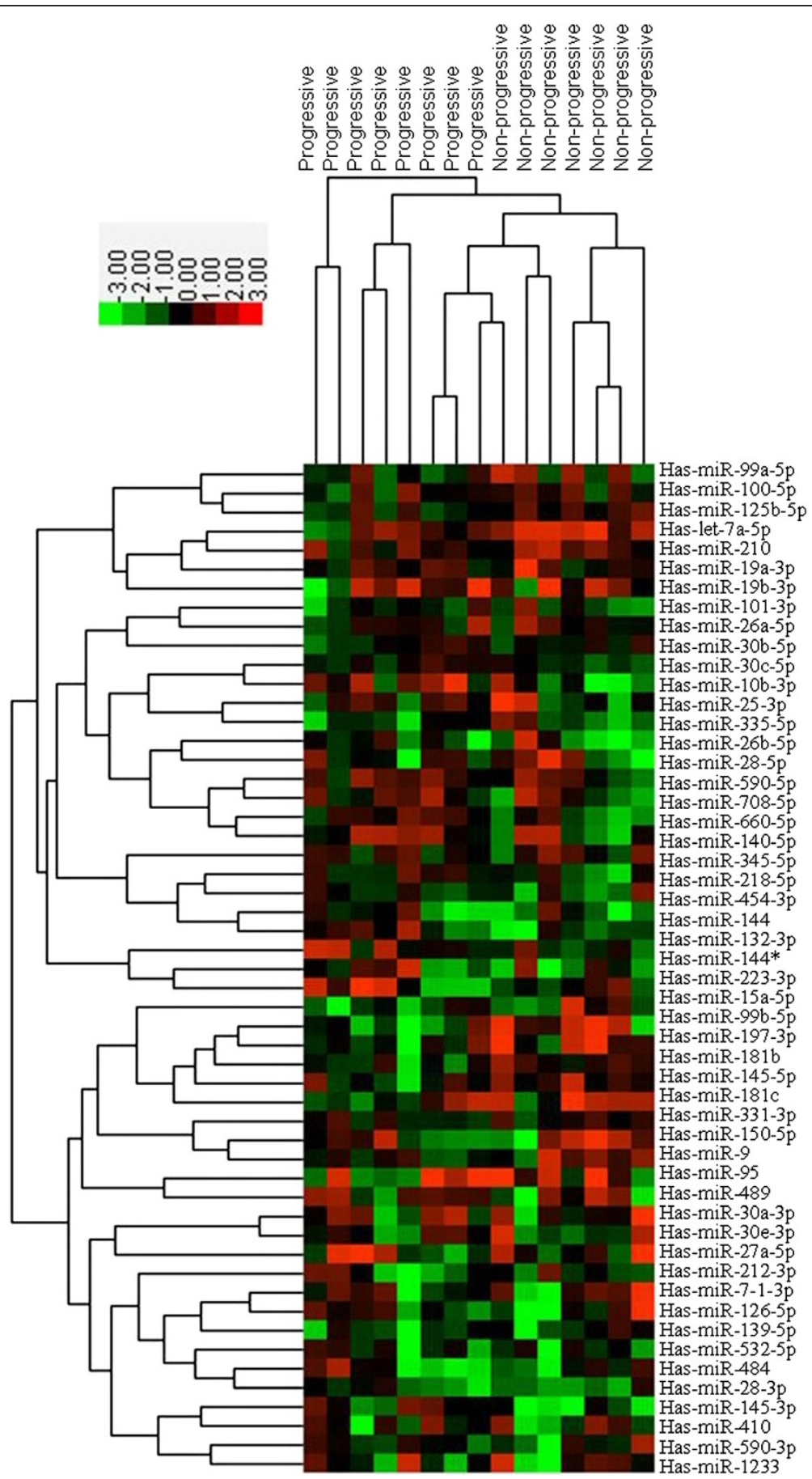

Figure 2 Differentially expressed miRNAs between progressive LGD leukoplakias and non-progressive LGD leukoplakias. Heat map showing the differentially expressed mirRNAs between progressing LGDs and non-progressing LGDs. Over-expressed miRNAs are colour-coded red, repressed miRNAs are colour-coded green. 
miRNAs were down-regulated and 12 miRNAs were up-regulated in progressive LGD leukoplakias. According to previous studies on oral squamous cell carcinomas, most of these deregulated miRNAs in progressive LGD leukoplakias were changed with same changing patterns as that in OSCC, such as down-regulation of miR-99, miR-let-7, miR-145 and up-regulation of miR-708, miR10b, miR-26a and miR-30e. Interestingly, in our study both miR-181c and miR-181b were found to be underexpressed in progressing LGD leukoplakias, which was contrary to the result of a previous study on miRNA expression changes during progression of oral premalignancy. The miRNAs that were differentially expressed are listed in Table 2 and Figure 2.

\section{Detection of deregulated miRNA expressions in saliva}

To explore the possibility of using salivary microRNA markers to distinguish OPL patients with different malignant transform potential, we tested the expression of 8 miRNAs (miR-10b, miR-145, miR-99b, miR-708, miR-181c, miR-30e, miR-660 and miR-197) in saliva samples from these patients using qRT-PCR. According to the TLDA findings, miR-10b, miR-660, miR-708 and miR-30e demonstrated significant over-expression in progressive LGD leukoplakias; miR-145, miR-99b, miR181c and miR-197 were under-expressed in this group of leukoplakias.

Expressions of miR-10b, miR-145, miR-99b, miR-708 and miR-181c were significantly different in saliva of progressive LGD leukoplakia patients, compared to that of non-progressive LGD leukoplakia patients (Figure 3). Trend $(\mathrm{P}<0.10)$ were observed for miR-197 and miR$30 \mathrm{e}$, the p-values were 0.057 and 0.089 respectively. Expression of miR-660 was under-expressed in nonprogressing LGD leukoplakias $(\mathrm{p}=0.038)$ but overexpression of this miRNA in progressing leukoplakias did not reach statistical significance $(P=0.098)$, which was inconsistent with our initial TLDA findings of this gene in progressing leukoplakias.

\section{Discussion}

Patients with oral premalignant changes consist of a diverse population and should be treated differently depending on their molecular genotype. There is an urgent need to identify which leukoplakia will progress and which will not. The search for genetic biomarkers of tumor progression is one of the potential approaches. miRNA expression profiling has been able to identify

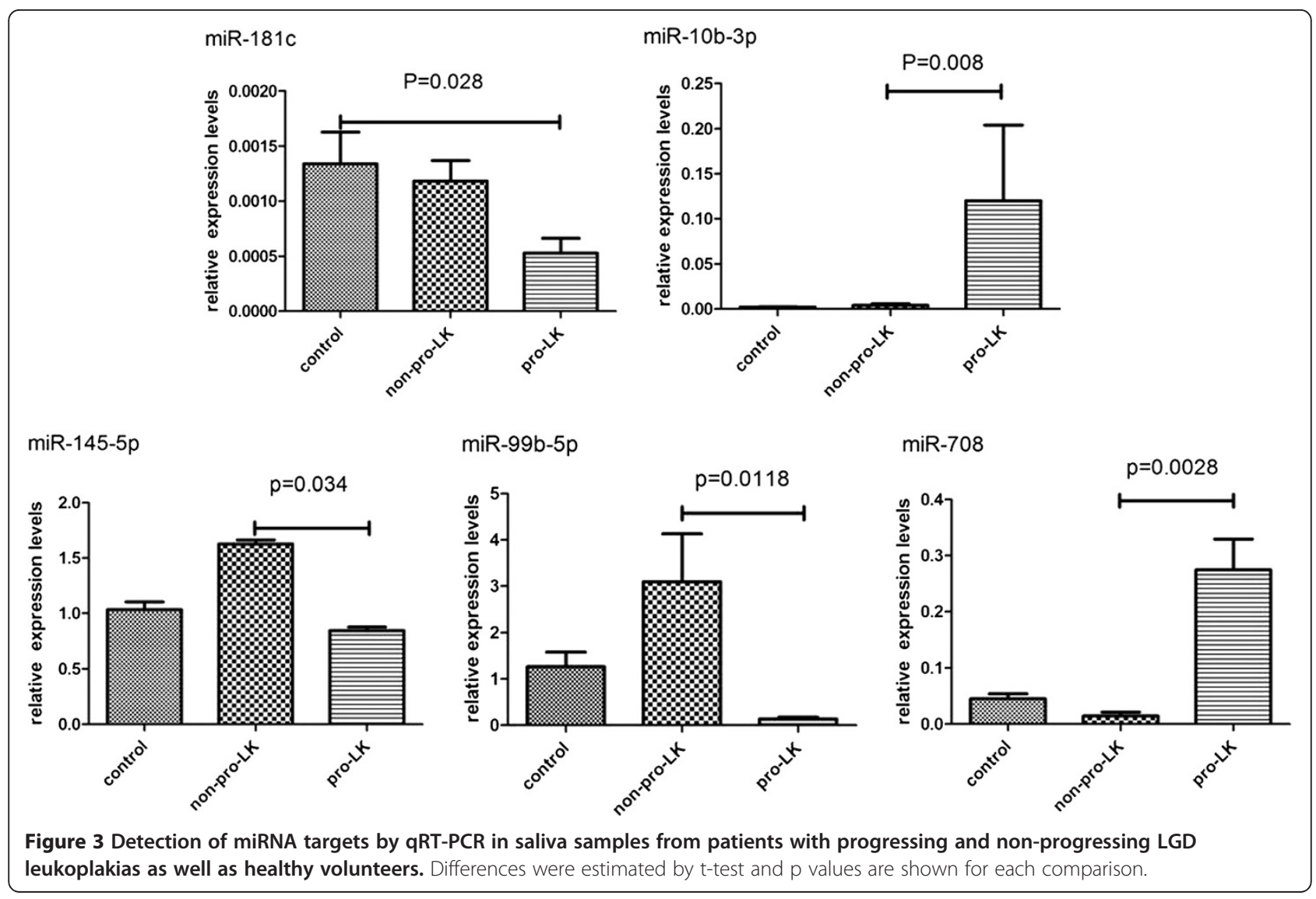


signatures associated with cancer initiation and progression. And abnormal changes in miRNA expression have been identified in patients' bodily fluids, such as blood, urine, and saliva $[18,19]$. In order to explore the possibility of salivary miRNA analysis as a method aiding in risk assessment of OPL, we examined miRNA expression in paired tissue and saliva samples from patients with histopathologically similar leukoplakias but different clinical outcomes. We successfully detected in OPL patients' saliva samples those miRNAs which were differentially expressed in OPL tissue samples. We found that saliva contains these deregulated miRNAs in much lower amounts, but they are present in this fluid and the changing patterns are similar with that in tissue samples.

In the present study we observed up-regulations of miR-708, miR-10b, miR-19a, miR-30e, miR-26a, miR-660 and down-regulations of miR-99, miR-15a, miR-197, miR-145 and miR-150. The same changing patterns have been reported in other cancers, including oral squamous cell carcinomas. However, we noticed miR-181c and miR$181 \mathrm{~b}$ were found to be under-expressed in progressing LGD leukoplakias and over-expressed in non-progressive LGD leukoplakias, which was contrary to the result of a previous study. The miR-181 family members were identified as either tumor suppressors or oncogenes in various cancers depending on tissue types [20-22]. Cervigne N.K. et al. reported that they found a gradual increase in expression of miR-181b in tissue samples during oral carcinogenesis, suggesting miR-181b plays as an oncogenic gene in oral cancer [15]. Our results were contrary to this but in concordance with results of some recent studies. For example, decreased serum miR-181a was found in breast cancer patients, especially early stage breast cancer patients (ductal carcinoma in situ, TNM I and II) [23]. Visone R. et al. found that during the course of the disease the expression of miR-181b was decreased in samples of patients with a progressive but not in samples of patients with a stable disease. It seems that miR181 family members may act as tumor suppressors in tumor initiation and decreased expression of these genes may contribute to progression from precursor lesions to tumors. Further studies needed to clarify this point.

We also noticed that a group of miRNAs are overexpressed in non-progressing LGD leukoplakias compared with progressing LGD leukoplakias. Among them are miR-197, miR-let-7, miR-99a/b, miR-126 and miR-145. These over-expressed miRNAs are known for their tumor suppressive roles in cancers. The marked up-regulation of these miRNAs in this group of patients implies that these stable LGD lesions may possess some mechanisms of protection from malignant transformation.

Several important conclusions can be made from the encouraging results of our study. Firstly, some miRNAs are differentially expressed in LGD leukoplakias with different progression potentials. Secondly, these differentially expressed miRNAs can be detected in both tissue samples and saliva samples as early as at LGD stage. Thirdly, Salivary miRNA analysis is a promising noninvasive assay for early detection and monitoring of oral premalignancies. But it should also be noted that our results were drawn from a small number of patients, as these progressing LGD leukoplakias are rare and very difficult to obtain. The different microRNA patterns are not intended to draw any disease specific conclusions and shown only as a proof of concept. However, when we compared the miRNA expression profiles of these LGD leukoplakias with different clinical outcomes, we did demonstrate a clear segregation between them and the similar expressions were detected in saliva samples of these patients. These preliminary results are suggestive to further develop this approach and the actual clinical value of this approach needs to be approved in larger cohort studies in high-risk groups.

\section{Conclusion}

Right now biopsy should still remain the gold standard in diagnosing oral precancer and oral cancer. However, detection of deregulated miRNA biomarkers in saliva samples is a promising noninvasive assay for risk assessment of LGD oral precancerous lesions, which may add value to the histologic diagnosis.

\section{Abbreviations \\ HGD: High grade dysplasia; LGD: Low grade dysplasia; CIS: Carcinoma in situ; OSCC: Oral squamous cell carcinomas; OPLs: Oral premaligant lesions; FDR: False discovery rate; TLDA: $\operatorname{TaqMan}{ }^{\circledR}$ low density array.}

\section{Competing interests}

The authors declare that they have no competing interests.

\section{Authors' contributions}

$Y Y$ and $Y Z$ were responsible for the study design, interpretation of the data and revision of the manuscript. $Y L$ and $X Y$ collected and processed the patients' samples, YY, LJ and ZZ carried out the experimental work the statistical analysis. YY prepared the manuscript, and YZ made critical revisions. All authors read and approved of the final manuscript.

\section{Acknowledgements}

This work was supported by research Grants 81000439 and 81271134 from National Natural Science Foundation of China.

\section{Author details}

'Department of General Dentistry, Ninth People's Hospital, School of Stomatology, Shanghai Jiao Tong University School of Medicine,Shanghai Key Laboratory of Stomatology, 639 Zhi Zao Ju Road, Shanghai 200011, China. ${ }^{2}$ Department of Stomatology, Tai'an Central Hospital, Tai'an, Shandong 271000, China. ${ }^{3}$ Department of Oral \& Maxillofacial-Head \& Neck Oncology, Ninth People's Hospital, School of Stomatology, Shanghai Jiao Tong University School of Medicine, Shanghai Key Laboratory of Stomatology and Shanghai Research Institute of Stomatology, Shanghai 200011, China.

Received: 26 December 2012 Accepted: 13 March 2013

Published: 19 March 2013 


\section{References}

1. Napier SS, Speight PM: Natural history of potentially malignant oral lesions and conditions: an overview of the literature. J Oral Pathol Med 2008, 37(1):1-10

2. Tsao AS, Kim ES, Hong WK: Chemoprevention of cancer. CA Cancer J Clin 2004, 54(3):150-180.

3. Wright JM: A review and update of oral precancerous lesions. Tex Dent $J$ 1998, 115(6):15-19.

4. Bouquot JE, Weiland LH, Kirland LT: Leukoplakia and carcinoma in situ synchronously associated with invasive oral/oropharyngeal carcinoma in Rochester, Minn., 1935-1984. Oral Surg Oral Med Oral Pathol 1988, 65(2):199-207.

5. Calin GA, Dumitru CD, Shimizu M, Bichi R, Zupo S, Noch E, Aldler H, Rattan S, Keating M, Rai K, Rassenti L, Kipps T, Negrini M, Bullrich F, Croce CM: Frequent deletions and down-regulation of micro-RNA genes miR15 and miR16 at 13q14 in chronic lymphocytic leukemia. Proc Natl Acad Sci 2002, 99(24):15524-15529.

6. Lu J, Getz G, Miska EA, Alvarez-Saavedra E, Lamb J, Peck D, Sweet-Cordero A, Ebert BL, Mak RH, Ferrando AA, Downing JR, Jacks T, Horvitz HR, Golub TR: MicroRNA expression profiles classify human cancers. Nature 2005, 435(7043):834-838.

7. O'Donnell KA, Wentzel EA, Zeller Kl, Dang CV, Mendell JT: c-Myc-regulated microRNAs modulate E2F1 expression. Nature 2005, 435(7043):839-843.

8. He L, Thomson JM, Hemann MT, Hernando-Monge E, Mu D, Goodson S, Powers S, Cordon-Cardo C, Lowe SW, Hannon GJ, Hammond SM: A microRNA polycistron as a potential human oncogene. Nature 2005, 435(7043):828-833.

9. Fabian MR, Sonenberg N, Filipowicz W: Regulation of mRNA Translation and Stability by microRNAs. Annu Rev Biochem 2010, 79:351-379.

10. Park NJ, Zhou H, Elashoff D, Henson BS, Kastratovic DA, Abemayor E, Wong DT: Salivary microRNA: discovery, characterization, and clinical utility for oral cancer detection. Clin Cancer Res 2009, 15(17):5473-5477.

11. Etheridge A, Lee I, Hood L, Galas D, Wang K: Extracellular microRNA: A new source of biomarkers. Mutat Res 2011, 717(1-2):85-90.

12. Mavrakis KJ, Wolfe AL, Oricchio E, Palomero T, de Keersmaecker K, McJunkin K, Zuber J, James T, Khan AA, Leslie CS, Parker JS, Paddison PJ, Tam W, Ferrando A, Wendel HG: Genome-wide RNA-mediated interference screen identifies miR-19 targets in Notch-induced T-cell acute lymphoblastic leukaemia. Nature Cell Biol 2010, 12(4):372-379.

13. Portela A, Esteller M: Epigenetic modifications and human disease. Nature Biotechnol 2010, 28(10):1057-1068.

14. Herceg Z, Paliwal A: Epigenetic mechanisms in hepatocellular carcinoma: how environmental factors influence the epigenome. Mutat Res 2011, 727(3):55-61.

15. Cervigne NK, Reis PP, Machado J, Sadikovic B, Bradley G, Galloni NN, Pintilie M, Jurisica I, Perez-Ordonez B, Gilbert R, Gullane P, Irish J, Kamel-Reid S: Identification of a microRNA signature associated with progression of leukoplakia to oral carcinoma. Hum Mol Genet 2009, 18(24):4818-4829.

16. Wiklund ED, Gao S, Hulf T, Sibbritt T, Nair S, Costea DE, Villadsen SB, Bakholdt V, Bramsen JB, Sørensen JA, Krogdahl A, Clark SJ, Kjems J: MicroRNA Alterations and Associated Aberrant DNA Methylation Patterns across Multiple Sample Types in Oral Squamous Cell Carcinoma. PLoS One 2011, 6(11):e27840.

17. Axell T, Pindborg JJ, Smith CJ, van der Waal I: Oral white lesions with special reference to precancerous and tobacco-related lesions: conclusions of an international symposium held in Uppsala, Sweden, May 18-21 1994. J oral path med 1996, 25(2):49-54.

18. von Brandenstein M, Pandarakalam JJ, Kroon L, Loeser H, Herden J, Braun G, Wendland K, Dienes HP, Engelmann U, Fries JW: MicroRNA 15a, inversely correlated to $\mathrm{PKCa}$, is a potential marker to differentiate between benign and malignant renal tumors in biopsy and urine samples. Am J Pathol 2012, 180(5):1787-1797.

19. Spielmann N, Wong DT: Saliva: diagnostics and therapeutic perspectives. Oral Dis 2011, 17(4):345-354.

20. Pekasky Y, Santanam U, Cimmino A, Palamarchuk A, Efanov A, Maximov V, Volinia S, Alder H, Liu CG, Rassenti L, Calin GA, Hagan JP, Kipps T, Croce CM: Tcl1 expression in chronic lymphocytic leukemia is regulated by miR-29 and miR-181. Cancer Res 2006, 66(24):11590-11593.

21. Iliopoulos D, Jaeger SA, Hirsch HA, Bulyk ML, Struhl K: STAT3 activation of miR-21 and miR-181b-1 via PTEN and CYLD are part of the epigenetic switch linking inflammation to cancer. Mol Cell 2010, 39(4):493-506.
22. Wang B, Hsu SH, Majumder S, Kutay H, Huang W, Jacob ST, Ghoshal K: TGFbeta-mediated upregulation of hepatic miR-181b promotes hepatocarcinogenesis by targeting TIMP3. Oncogene 2010, 29(12):1787-1797.

23. Visone R, Veronese A, Rassenti LZ, Balatti V, Pearl DK, Acunzo M, Volinia S, Taccioli C, Kipps TJ, Croce CM: miR-181b is a biomarker of disease progressiong in chronic lymphocytic leukemia. Blood 2011, 118(11):3072-3079.

doi:10.1186/1471-2407-13-129

Cite this article as: Yang et al:: Progress risk assessment of oral premalignant lesions with saliva miRNA analysis. BMC Cancer 2013 13:129.

\section{Submit your next manuscript to BioMed Central and take full advantage of:}

- Convenient online submission

- Thorough peer review

- No space constraints or color figure charges

- Immediate publication on acceptance

- Inclusion in PubMed, CAS, Scopus and Google Scholar

- Research which is freely available for redistribution

Submit your manuscript at www.biomedcentral.com/submit
C BioMed Central 